\title{
Kinetics and Energetics of Oligomer Desorption from Surfaces
}

\author{
Kris R. Paserba and Andrew J. Gellman* \\ Department of Chemical Engineering, Carnegie Mellon University, Pittsburgh, Pennsylvania 15213
}

(Received 30 November 2000)

\begin{abstract}
The dynamics of oligomer desorption from surfaces has been studied by measuring the desorption kinetics of a set of straight chain alkanes $\left[\mathrm{H}\left(\mathrm{CH}_{2}\right)_{n} \mathrm{H}\right.$, with $n=5$ to 60] from the surface of single crystalline graphite. Desorption is observed to be a first-order process and the preexponent of the desorption rate constant has a value $\nu=10^{19.6 \pm 0.5} \mathrm{sec}^{-1}$ and is independent of the oligomer chain length. More interestingly, we find that the barrier to desorption has a nonlinear dependence on chain length and takes the form $\Delta E_{\mathrm{des}}^{\ddagger}=A+B n^{\alpha}$, with the exponent $\alpha=0.50 \pm 0.01$.
\end{abstract}

DOI: $10.1103 /$ PhysRevLett. 86.4338

This Letter reports the barriers to desorption $\left(\Delta E_{\mathrm{des}}^{\ddagger}\right)$ of straight chain alkanes $\left[\mathrm{H}\left(\mathrm{CH}_{2}\right)_{n} \mathrm{H}\right.$, with $n=5$ to 60] from the surface of graphite into vacuum and the finding of a nonlinear dependence of $\Delta E_{\mathrm{des}}^{\ddagger}$ on chain length, $n$. This nonlinear behavior reveals something of the dynamics of the complex process or mechanism by which oligomeric species desorb from surfaces. Independent of its fundamental interest, this result has implications for a number of technologically important surface phenomena such as the evaporation of thin lubricant films from the surfaces of magnetic data storage disks and the desorption of alkanes from the surfaces of Fischer-Tropsch catalysts.

The vast majority of measurements of molecular desorption kinetics from surfaces have used relatively small species for which the desorption process is considered simply as a displacement along the surface normal. This desorption mechanism can be adequately modeled using a single well-defined reaction coordinate through a fairly simple potential energy surface describing the interaction of the molecule with the surface. The desorption rate constant is usually considered to have the form given by the transition state theory [1]

$$
k=\left(\frac{k_{B} T}{h}\right) \frac{q_{\ddagger}}{q} \exp \left(\frac{-\Delta E_{\mathrm{des}}^{\ddagger}}{k_{B} T}\right),
$$

where $h$ is Planck's constant and $k_{B}$ is Boltzmann's constant. The $\Delta E_{\text {des }}^{\ddagger}$ is the difference between the zero-point energies of the adsorbed state and the transition state for desorption. At best the multidimensional nature of the adsorbate-substrate potential energy surface influences the desorption kinetics through the partition functions for the adsorbed state $(q)$ and the transition state for desorption $\left(q_{\ddagger}\right)$.

Consider the desorption of an oligomeric species such as $\mathrm{H}\left(\mathrm{CH}_{2}\right)_{60} \mathrm{H}$ from a surface. Many scanning tunneling micrographs (STM) of alkanes adsorbed on graphite at room temperature reveal an all-trans conformation stretched out and interacting with the surface along its length [2-5]. Since the desorbed state has no segments interacting with the surface one might predict that the $\Delta E_{\mathrm{des}}^{\ddagger}$ should be linear in the chain length. There have been several prior sets of measurements which observe the effects of alkyl chain
PACS numbers: $68.43 . \mathrm{Vx}$

length on the desorption kinetics of species such as alkyl alcohols and simple alkanes adsorbed on metal surfaces [6-9]. In these cases the range of alkyl chain lengths has been limited to $n \leq 12$. Needless to say, over this limited range the measured values of $\Delta E_{\mathrm{des}}^{\ddagger}$ are linear in $n$. However, one can imagine a multitude of energetically equivalent trajectories by which an oligomer such as $\mathrm{H}\left(\mathrm{CH}_{2}\right)_{60} \mathrm{H}$ might desorb from a surface. The combined contributions of these trajectories must influence the overall desorption reaction kinetics. In this Letter we report the results of the first set of measurements to use a broad enough set of oligomer chain lengths, $n=5$ to 60 , to allow observation of a nonlinear dependence of $\Delta E_{\text {des }}^{\ddagger}$ on $n$.

Adsorption and desorption of the straight chain alkanes from graphite were performed under ultrahigh vacuum (UHV) conditions. The sample of highly oriented pyrolytic graphite was mounted on a UHV manipulator that allowed cooling to $100 \mathrm{~K}$ and heating to $1000 \mathrm{~K}$. The graphite surface was prepared simply by peeling off the top layers in air and then cleaned by heating to $1000 \mathrm{~K}$ following its introduction into the vacuum chamber. This procedure was deemed sufficient since the desorption kinetics of the short alkanes from the surface were extremely reproducible. During a normal temperature programmed desorption experiment using heptane $\left(\mathrm{C}_{7} \mathrm{H}_{16}\right)$ the desorption temperature was always found to be $211 \mathrm{~K}$ and the width of the desorption peak was $\leq 7 \mathrm{~K}$. Adsorption of the alkanes was always performed with the graphite surface at $<120 \mathrm{~K}$. For the alkanes with $n \leq 12$ adsorption was performed using a leak valve to introduce the vapor into the vacuum chamber at a controlled pressure for a controlled period of time. The feature of our apparatus that allowed experiments with long chain alkanes is a dosing system developed to allow the introduction of species with extremely low vapor pressures. This device will be described elsewhere but basically consists of a heated zone in which the species of interest is vaporized and a long collimating tube which can be positioned directly in front of the graphite sample or directly in front of the aperture to the mass spectrometer [10]. The 21 alkanes with $n$ in the range 5 through 60 were all purchased from commercial sources. Although 
these are nominally monodispersed as provided the higher molecular weight species required extensive degassing to remove low molecular weight, high vapor pressure components to the point that the primary species coming from the doser was the species of choice. Once purified the high molecular weight alkanes were adsorbed on the graphite surface by positioning the sample immediately in front of the collimating tube of the doser with the heated zone at a temperature in the range 40 to $250{ }^{\circ} \mathrm{C}$ depending upon the alkane being used. The coverage of alkane on the surface was controlled by the time of exposure. Once the alkane was adsorbed at the desired coverage the graphite surface was positioned in front of a quadrupole mass spectrometer and heated at a constant rate from 130 to $850 \mathrm{~K}$ in order to induce desorption of the alkane. For each of the 21 alkanes these thermal desorption experiments were performed with a heating rate of $2 \mathrm{~K} / \mathrm{s}$ and coverages ranging from $<0.1$ to $>1$ monolayers. For a subset of nine alkanes the desorption experiments were also performed using a constant coverage of 1 monolayer and variable heating rates in the range $0.1-5 \mathrm{~K} / \mathrm{s}$. In all instances the desorption of the alkane is molecular. There is only a single species desorbing from the surface over a narrow temperature range and there is no evidence for decomposition of the adsorbed species or contamination of the surface by fragments still adsorbed after heating to $850 \mathrm{~K}$.

Thermal desorption spectra were obtained for all 21 alkanes as a function of coverage on the graphite surface. The desorption spectra for $\mathrm{C}_{32} \mathrm{H}_{66}$ are illustrated in Fig. 1 and are representative of those observed for all the alkanes. At low exposures there is a single desorption peak at $523 \mathrm{~K}$ that grows with increasing coverage. This high temperature peak is due to the desorption of the $\mathrm{C}_{32} \mathrm{H}_{66}$ monolayer from the graphite surface. For the shorter chain alkanes this monolayer desorption peak saturates in intensity as the coverage increases. As it approaches saturation a second set of desorption peaks appears at lower temperature $\left(350 \mathrm{~K}\right.$ for $\left.\mathrm{C}_{32} \mathrm{H}_{66}\right)$ which is due to desorption of the multilayer from the surface. The double peak structure observed in the multilayer desorption of $\mathrm{C}_{32} \mathrm{H}_{66}$ is consistently observed for all the longer chain alkanes. Although its origin is not known it is possible that this is due to desorption from the second and then the third alkane layers on the graphite surface. The important result revealed by Fig. 1 is that the monolayer desorption feature has a peak temperature which is coverage independent. This is consistent with a first-order desorption process having a desorption barrier that is independent of coverage, in other words, a desorption rate equation of the form

$$
r=\nu \exp \left(\frac{-\Delta E_{\mathrm{des}}^{\ddagger}}{k_{B} T}\right) \theta
$$

where $\theta$ is the coverage of adsorbed alkane and $\nu$ is a preexponential factor.

The fact that all the alkanes desorb from the graphite surface with such simple and well-defined kinetics means

\section{TPD - $\mathrm{C}_{32} \mathrm{H}_{66} /$ Graphite}

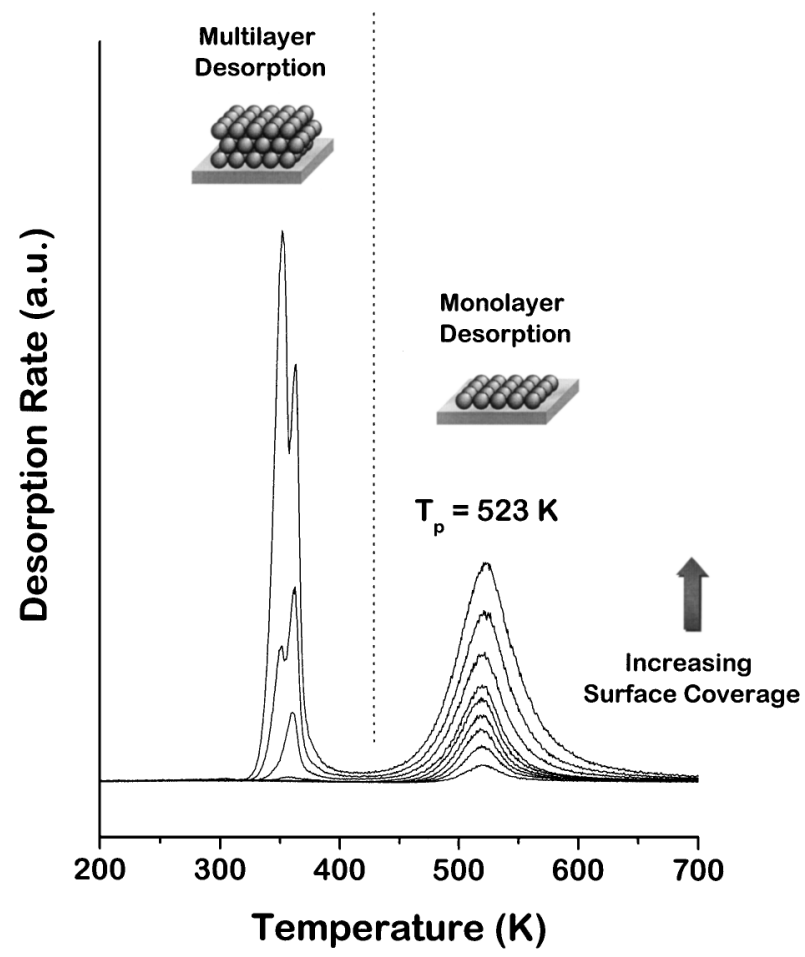

FIG. 1. Thermally programmed desorption spectra for $\mathrm{C}_{32} \mathrm{H}_{66}$ adsorbed on the surface of graphite at $120 \mathrm{~K}$ at various coverages. The desorption peak at $523 \mathrm{~K}$ is due to the monolayer adsorbed directly to the graphite surface. The features at $350 \mathrm{~K}$ are assigned to desorption from the multilayers. The monolayer desorption temperature is independent of coverage indicating a first-order desorption process with a coverage independent desorption barrier. The heating rate was $2 \mathrm{~K} / \mathrm{s}$ and the ion fragment monitored with the mass spectrometer was at $m / q=57\left(\mathrm{C}_{4} \mathrm{H}_{9}{ }^{+}\right)$.

that the desorption spectra can be analyzed to obtain kinetic parameters. The simplest analysis is the Redhead method which relates the desorption kinetic parameters $\nu$ and $\Delta E_{\mathrm{des}}^{\ddagger}$ to the experimental variables $\beta$ (the heating rate) and $T_{p}$ (the peak desorption temperature) through the equation [11]

$$
\ln \left(\frac{\beta}{T_{p}^{2}}\right)=\ln \left(\frac{k_{B} \nu}{\Delta E_{\mathrm{des}}^{\ddagger}}\right)-\frac{\Delta E_{\mathrm{des}}^{\ddagger}}{k_{B} T_{p}} .
$$

By measuring $T_{p}$ using a range of heating rates it is possible to obtain $\nu$ and $\Delta E_{\mathrm{des}}^{\ddagger}$ independently. Variable heating rate desorption experiments have been performed using a set of nine alkanes in the length range $n=7$ to 44 . The values of the desorption preexponent $\nu$ obtained from these variable heating rate experiments are plotted in Fig. 2 as a function of $n$. The desorption preexponent appears to be roughly independent of chain length with a value of $\nu=10^{19.6 \pm 0.5} \mathrm{sec}^{-1}$. The fact that this is substantially higher than the value of $k_{B} T / h\left(\sim 10^{13} \mathrm{sec}^{-1}\right)$ indicates that the partition function for the transition state to desorption is much higher than that of the adsorbed alkane. 


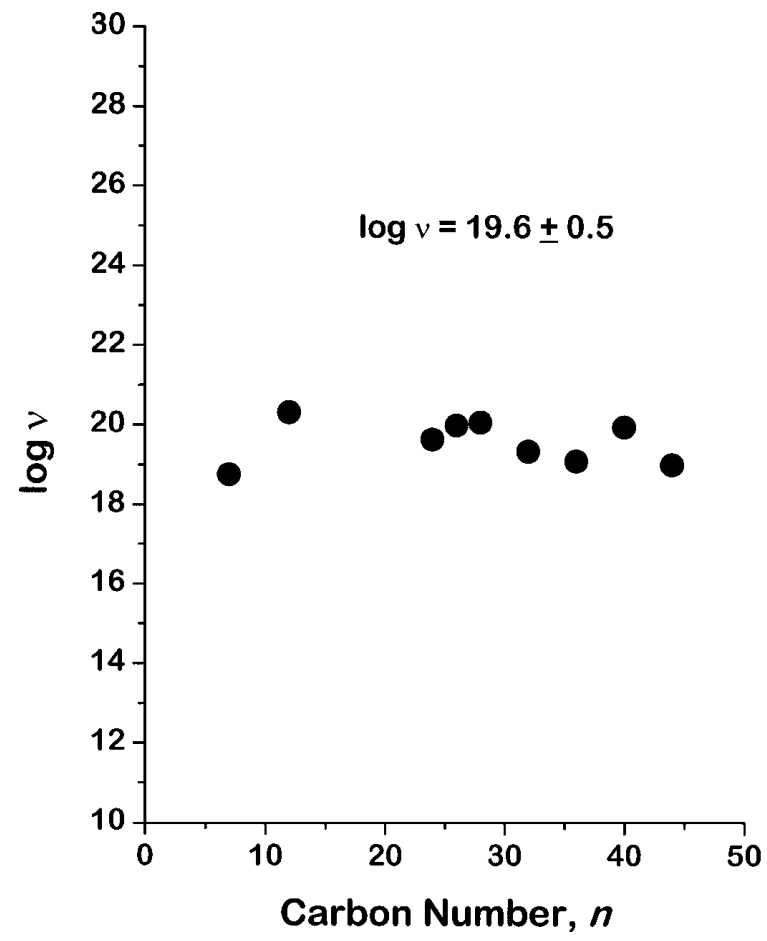

FIG. 2. The preexponential factors $(\nu)$ of the first-order desorption rate constants $(k)$ for alkanes of varying chain length ( $n=7$ to 44 ). These were determined from thermal desorption spectra obtained at varying heating rates $(\beta=0.1$ to $5 \mathrm{~K} / \mathrm{sec})$. The value of $\log (\nu)$ is apparently independent of alkane chain length.

This can be ascribed to a transition state with relatively free lateral translational motion across the surface [1]. In long straight chain alkanes there are many degrees of freedom that can, in principle, become free once portions of the chain are desorbed from the surface. These degrees of freedom might influence the desorption preexponent although one would expect such effects to be dependent on the chain length. The fact that the desorption preexponent is independent of chain length suggests that its magnitude is indeed due to a degree of freedom common to all the alkanes such as center of mass translation in the transition state.

Desorption spectra measured at varying alkane coverages using a constant heating rate have been obtained for all 21 straight chain alkanes used in this investigation. Using a desorption preexponent of $\nu=10^{19.6} \mathrm{sec}^{-1}$ we have determined the $\Delta E_{\mathrm{des}}^{\ddagger}$ for each alkane based on the peak desorption temperatures $T_{p}$, which lie in the range 169 to $726 \mathrm{~K}$. The values of $\Delta E_{\text {des }}^{\ddagger}$ are plotted in Fig. 3 and reveal the nonlinear dependence on chain length, $n$. As a first approach to the analysis of the nonlinear dependence of $\Delta E_{\mathrm{des}}^{\ddagger}$ on chain length we have fit the data to a power law expression of the form $\Delta E_{\mathrm{des}}^{\ddagger}=A+B n^{\alpha}$. The reason for including the offset $A$ rather than using pure power law scaling $\left(\propto n^{\alpha}\right)$ is that the ends of the alkane chains are unlikely to have the same interaction with the surface as the chain segments in the middle of the molecule. For

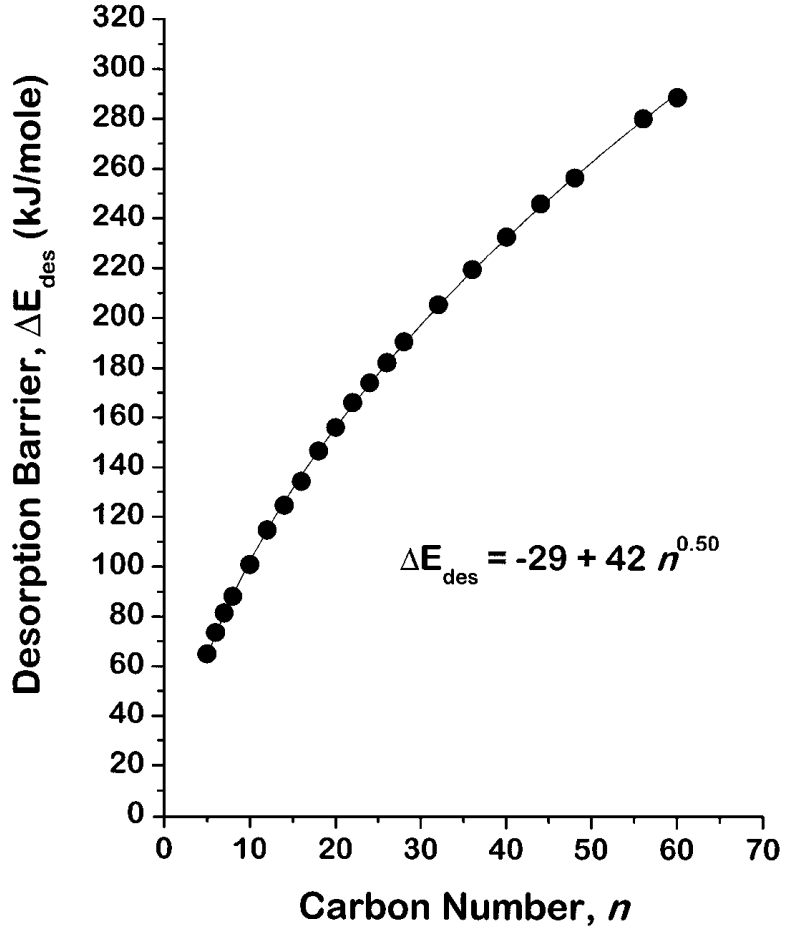

FIG. 3. The barriers to desorption $\left(\Delta E_{\mathrm{des}}^{\ddagger}\right)$ for alkanes with $n=5$ to 60 adsorbed on the surface of graphite. These were determined from peak desorption temperatures that ranged from 169 to $726 \mathrm{~K}$ by using a first-order desorption rate law and a value of the desorption preexponent of $\nu=10^{19.6} \mathrm{sec}^{-1}$. The range of $n$ is clearly large enough to reveal the nonlinearity of the $\Delta E_{\mathrm{des}}^{\ddagger}$. The solid curve through the data is a fit to the offset power law $\Delta E_{\mathrm{des}}^{\ddagger}=A+B n^{\alpha}$ and reveals a power-law dependence of $\alpha=0.50 \pm 0.01$.

small alkanes this will certainly be a significant contribution to the desorption energy and the offset $A$ is a correction for that effect. The fitting parameters found for the $\Delta E_{\text {des }}^{\ddagger}$ dependence on chain length are $A=-29 \pm 4 \mathrm{~kJ} / \mathrm{mole}$, $B=42 \pm 2$, and an exponent of $\alpha=0.50 \pm 0.01$. It is interesting to note that the extrapolation of the expression for $\Delta E_{\mathrm{des}}^{\ddagger}$ yields a value of $13 \mathrm{~kJ} / \mathrm{mole}$ for the desorption of methane from graphite which is extremely close to the well-determined value of $E_{b}=12.2 \mathrm{~kJ} / \mathrm{mole}$ reported for the binding energy of methane on graphite [12]. This close comparison may be fortuitous since the extrapolated value of $\Delta E_{\mathrm{des}}^{\ddagger}=30 \mathrm{~kJ} / \mathrm{mole}$ for ethane does not match as closely the experimentally, although less welldetermined, value of $E_{b}=17.2 \mathrm{~kJ} / \mathrm{mole}$. Nonetheless the fit for $n=5$ to 60 reproduces the data extremely well and is shown as the solid line in Fig. 3.

In view of the fact that there are many room temperature images of the alkanes on graphite which show these molecules adsorbed in straight, all-trans configurations on the surface, it is extremely interesting that the $\Delta E_{\text {des }}^{\ddagger}$ do not scale linearly in chain length [2-5]. The implication is that at the desorption temperature configurational entropy in either the initial state or the transition state to desorption decreases the net $\Delta E_{\mathrm{des}}^{\ddagger}$ for the longer chain 
oligomers. The possibility that this entropy increase occurs in the initial state is borne out by variable temperature STM studies that reveal reversible disordering of the alkanes at temperatures above $300 \mathrm{~K}$ [2-5]. The fact that $\left(\Delta E_{\mathrm{des}}^{\ddagger}-A\right)$ scales as $n^{1 / 2}$ is also intriguing. It should be noted that a true power-law representation of $\log \left(\Delta E_{\text {des }}^{\ddagger}\right)$ versus $\log (n)$ yields a curve with a slope of 0.65 at $n=5$ that tends to 0.55 at $n=60$. These results suggest that as a result of configurational entropy the number of monomers interacting with the surface at the desorption temperature is of order $n^{1 / 2}$ rather than $n$.

There are many theoretical and some experimental studies of the behavior of polymeric species interacting with rigid surfaces. These indicate that under certain conditions the fraction of segments of an adsorbed polymer of length $n$ that are attached to the surface scales as $n^{1 / 2}$. The theoretical description of polymer adsorption at surfaces is in terms of structures having "trains" of sequential segments adsorbed to the surface, "loops" of desorbed segments between the trains, and desorbed "tails" at either end. Early descriptions based on random walks and Monte Carlo simulations modeled the case of the isolated chain at a surface $[13,14]$. These showed that the fraction of adsorbed segments for a polymer of length $n$ is proportional to $n^{1 / 2}$ for critical values of the segmentsurface interaction energy that are of order $k_{B} T$. For other values of the interaction energy the scaling constant tends towards $\frac{1}{2}$ for low values of $n$. At the interface between a solid surface and a polymer melt the fraction of segments of a given polymer molecule attached to the surface scales as $n^{1 / 2}$ [15]. Experimental and theoretical studies of the detachment rates of polymers from surfaces into solution and into a melt also show power-law scaling with chain length [16,17]. The experimental measurement of detachment rates into a polymer melt revealed a friction force against a strongly interacting surface that scales as $n^{1 / 2}$. In all there are a number of observations that suggest that interaction strengths of polymers with surfaces and detachment rates can scale with $n^{1 / 2}$. Although the results of our experiments certainly suggest this type of behavior, it is not clear that the conditions of our measurement correspond to those of previous such observations.

The measurements of $\Delta E_{\mathrm{des}}^{\ddagger}$ reported in this Letter are for alkane oligomers adsorbed at a graphite-vacuum interface. The ability to vary temperature over a wide range has enabled us to measure desorption rate constants that would vary by almost 40 orders of magnitude at room temperature. In terms commonly used to discuss polymersurface systems ours might be described as desorption from a strongly interacting surface into an infinitely dilute, poor solvent (vacuum). The fact that the desorption kinetics that we measure are first order in coverage suggests that one can consider the molecules as isolated in the sense that intermolecular interactions are weak by comparison with alkane-graphite interactions. Modeling of these results that is to be described elsewhere suggests that the segment-surface interaction energy is $\varepsilon=$ $8.2 \mathrm{~kJ} / \mathrm{mole}$ [10]. This means that the segment-surface interaction energy at the desorption temperature ranged from $\varepsilon=5.8 k_{B} T$ [for $\mathrm{H}\left(\mathrm{CH}_{2}\right)_{5} \mathrm{H}$ ] to $\varepsilon=1.4 k_{B} T$ [for $\left.\mathrm{H}\left(\mathrm{CH}_{2}\right)_{60} \mathrm{H}\right]$. These are the first such measurements of their type and have measured $\Delta E_{\text {des }}^{\ddagger}$ for a very simple and well-defined oligomer-surface system. Although there is plenty of evidence that suggests $n^{1 / 2}$ scaling for related systems and similar properties, the rationale for this scaling of $\Delta E_{\text {des }}^{\ddagger}$ under the conditions of our measurement is still not clear.

Support from the NSF (CMS-9900647) is acknowledged for this work. The development of the high molecular weight doser was supported by the AFOSR (F49620-96-10253).

*Author to whom correspondence should be addressed.

[1] V. P. Zhdanov, Surf. Sci. Rep. 12, 183 (1991).

[2] L. Askadskaya and J. P. Rabe, Phys. Rev. Lett. 69, 1395 (1992).

[3] J.-P. Bucher, H. Roeder, and K. Kern, Surf. Sci. 289, 370 (1993).

[4] R. Hentschke, B. L. Schürmann, and J. P. Rabe, J. Chem. Phys. 96, 6213 (1992).

[5] G. Watel, F. Thibaudau, and J. Cousty, Surf. Sci. Lett. 281, L297 (1993).

[6] R. Zhang and A.J. Gellman, J. Phys. Chem. 95, 7433 (1991).

[7] S. M. Wetterer, D. J. Lavrich, T. Cummings, S. L. Bernasek, and G. Scoles, J. Phys. Chem. 102, 9266 (1998).

[8] A. V. Teplyakov, A. B. Gurevich, M. X. Yang, B. E. Bent, and J. G. Chen, Surf. Sci. 396, 340 (1998).

[9] J. L. Brand, M. V. Arena, A. A. Deckert, and S. M. George, J. Chem. Phys. 92, 5136 (1990).

[10] K. R. Paserba and A. J. Gellman (to be published).

[11] P. A. Redhead, Vacuum 12, 203 (1962).

[12] G. Vidali, G. Ihm, H.-Y. Kim, and M. W. Cole, Surf. Sci. Rep. 12, 133 (1991).

[13] E. A. Dimarzio and F. L. McCrackin, J. Chem. Phys. 43, 539 (1965).

[14] R.-J. Roe, J. Chem. Phys. 43, 1591 (1965).

[15] I. A. Bitsanis and G. ten Brinke, J. Chem. Phys. 99, 3100 (1993).

[16] Y. Wang and R. Rajagopalan, J. Chem. Phys. 105, 696 (1996).

[17] X. Zheng, B. B. Sauer, J. G. Van Alsten, S. A. Schwarz, M. H. Rafailovich, J. Sokolov, and M. Rubinstein, Phys. Rev. Lett. 74, 407 (1995). 